\title{
Nano-scale Elastic Strain Maps of Twins in Magnesium Alloys
}

\author{
Luoning Ma ${ }^{1}$, Paul F. Rottmann ${ }^{2}$ Kelvin Xie ${ }^{1,3}$, Kevin J. Hemker ${ }^{1}$ \\ 1. Department of Mechanical Engineering, Johns Hopkins University, Baltimore, MD, USA \\ 2. Materials Department, University of California, Santa Barbara, Santa Barbara, CA, USA \\ 3. Materials Science and Engineering Department, Texas A\&M, College Station, TX, USA
}

The nucleation and propagation mechanisms of extension twin boundaries (TB) have aroused great experimental and computational interests [1]. It is commonly accepted that the propagation of deformation twins in magnesium is attributed to the glide of twinning dislocations or disconnections (TD) on the twin plane $\left(\mathrm{K}_{1}\right)$ along the twinning direction $\left(\eta_{1}\right)$, which is activated by a combination of shear stress and atomic shuffling $[2,3]$. However, it has been frequently observed that the TBs of $\{-1012\}$ twins deviate from the ideal $\mathrm{K}_{1}$ planes, especially at the tips $[4,5]$. Braisaz, et al. noted that the abnormal deformation around the twin tips can be accommodated by the combination of regular $\mathrm{K}_{1}$ boundaries as well as a high-angle basal-prismatic (BP/PB) boundary [6]. They also pointed out that the TDs can slip on $\mathrm{K}_{1}$ boundaries and cross-slip onto the $\mathrm{BP} / \mathrm{PB}$ boundaries to propagate the twins. On the other hand, $\mathrm{Li}$ and Ma proposed a shuffling model based on their MD simulations to explain the propagation of twins without the help of TDs [7]. Nevertheless, the shuffling model remains controversial because it contradicts the well-accepted conservative principle of twin propagation.

The failure of conserving the $\mathrm{K}_{1}$ twin plane at the twin tip can be expected to generate stress concentrations no matter whether the TD glide mechanism dominates or the shuffling mechanism. And the characterization of the strain/stress field around the twin tips can in turn help analyze the propagation mechanism of extension twins. In this work, the following questions related to the strain field at the tips of extension twins in magnesium alloy have been addressed: i) what the magnitude of the elastic strain around a twin tip is; ii) whether stress is generated in the grains with twin formation and propagation; and iii) what the interaction between twin tips and grain boundaries or other defects in the alloys is?

To start to address the aforementioned questions, a novel technique, TOPSPIN, is being utilized to experimentally measure the elastic strain field around twin tips that were generated by compressing AZ31B magnesium alloy along a axis. The NanoMEGAS ASTAR system employs precession electron diffraction (PED) and automated indexing to create orientation and phase maps with nanoscale spatial resolution[8], and AppFive recently teamed with NanoMEGAS to develop an "AutoSTRAIN acquisition $\&$ analysis module" that facilitates high-precision elastic strain mapping with a reported precision of $2 \mathrm{x}$ $10^{-4}[9]$. To measure the relative strain level around twin tip, we first captured the diffraction pattern from a defect-free area and used that as the reference. Pixel-by-pixel acquisition of diffraction patterns in the area of interest, e.g. around the twin tips, and comparison with the reference facilitates the strain mapping with nanometer scale spatial resolution. Using this technique, we were able to measure the strain field around twin tips and inside grains, as well as the strain field around the junction of a twin and grain boundary. Figure 1 shows an example of the strain concentration that forms at a twin tip that is embedded inside of a grain. Figure 2 shows the tensile strain field in the adjacent grain at the junction of twin tip and grain boundary.

In summary, these preliminary results provide experimental evidence for the concentration of elastic strains and stresses at twin tips in magnesium alloys. That strain can be released by the formation and 
motion of basal dislocations, and when the twin is arrested at a grain boundary, an elastic strain field is generated in the adjacent grain. The extension of strain mapping from semiconductor devices is in its infancy, but mechanistic examples like this appear very promising.

\section{References:}

[1] J. Wang, I.J. Beyerlein, J.P. Hirth, Model Simul Mater Sc, 20 (2012).

[2] J.W. Christian, S. Mahajan, Prog Mater Sci, 39 (1995), p. 1.

[3] J. Wang, I.J. Beyerlein, J.P. Hirth, C.N. Tome, Acta Mater, 59 (2011), p. 3990.

[4] B. Li, X.Y. Zhang, Scripta Mater, 71 (2014), p. 45.

[5] X.Y. Zhang, et al., Scripta Mater, 67 (2012), p. 862.

[6] T. Braisaz, P. Ruterana, G. Nouet, Philos Mag A, 76 (1997), p. 63.

[7] B. Li, E. Ma, Phys Rev Lett, 103 (2009).

[8] E.F. Rauch, M. Veron, Materialwiss Werkst, 36 (2005), p. 552.

[9] D. Viladot, et al., Journal of Microscopy, 252 (2013), p. 23.
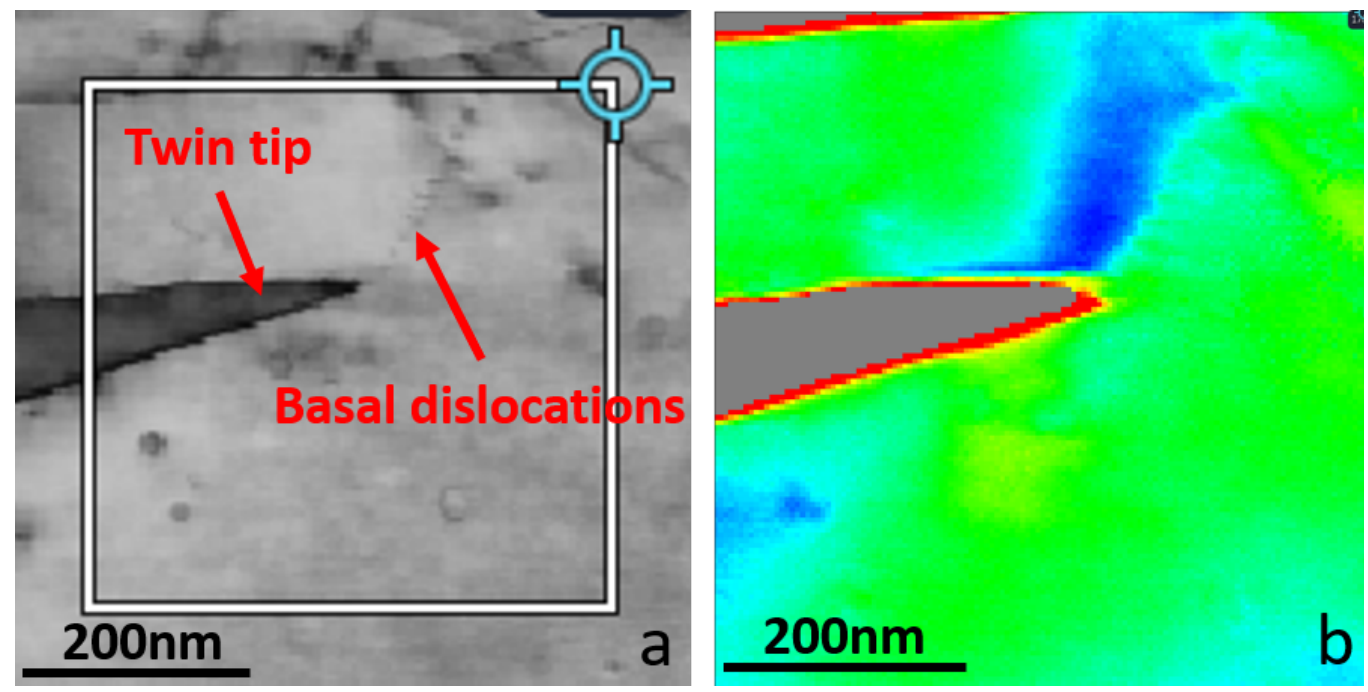

Figure 1. virtual bright field of an embedded twin (a) and the strain field around the tip in the grain (b).
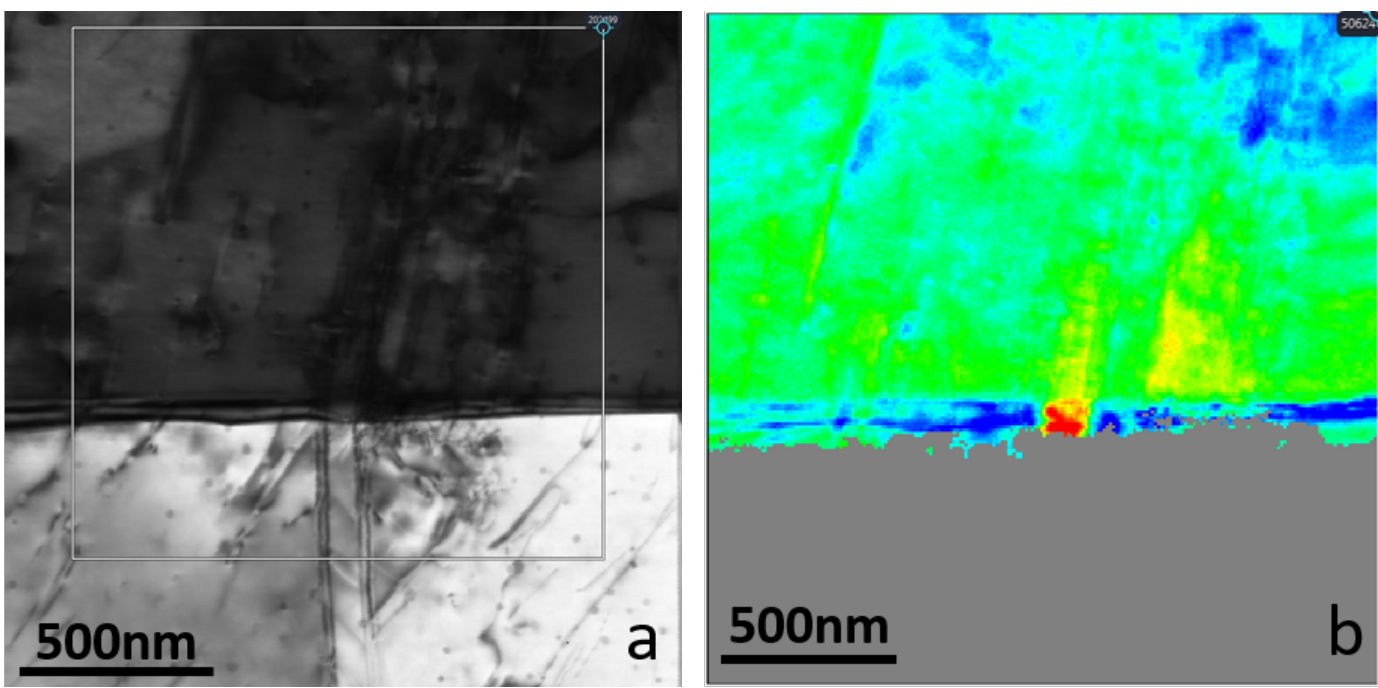

Figure 2. The strain field in a neighboring grain at the junction of twin tip and a grain boundary. 